\title{
Heterogeneity Gap in Stable Jurisdiction Structures
}

\author{
Anna Bogomolnaia* $\quad$ Michel Le Breton ${ }^{\dagger} \quad$ Alexei Savvateev $^{\ddagger} \quad$ Shlomo Weber $^{\S}$
}

March 2006

\begin{abstract}
This paper examines a model of multi-jurisdiction formation where individuals' characteristics are uniformly distributed over a finite interval. Every jurisdiction locates a public facility and distributes its cost equally among the residents. We consider two notions of stability: Nash stability and its refinement local Nash stability, and examine the existence and characterization of stable partitions. The main feature of this analysis is that, even under the uniform distribution, there are stable structures that exhibit a high degree of heterogeneity of jurisdiction sizes.
\end{abstract}

Keywords: Stability, Jurisdictions, Public Projects, Heterogeneity Gap.

JEL Classification Numbers: D70, H20,H73.

${ }^{*}$ Rice University, Houston, USA.

${ }^{\dagger}$ Université de Toulouse I, GREMAQ and IDEI, Toulouse, France.

${ }^{\ddagger}$ New Economic School, Moscow; Central Economics and Mathematics Institute, Moscow, Institute for Theoretical and Experimental Physics, Moscow; CORE, Catholic University of Louvain-la-Neuve, Belgium. Financial support through grants R98-0631 from the Economic Education and Research Consortium, \# NSh-1939.2003.6 School Support, Russian Foundation for Basic Research No. 04-02-17227, and the Russian Science Support Foundation is gratefully acknowledged.

${ }^{\S}$ Southern Methodist University, Dallas, USA; CORE, Catholic University of Louvain-la-Neuve, Belgium; and CEPR 


\section{Introduction}

The main objective of this paper is to explore the question of jurisdiction formation for a specific class of environments. ${ }^{1}$ Like any model of jurisdiction formation, its ultimate goal is to explain how a given homogeneous or heterogeneous population of economic units (individuals, cities, regions) divides itself into several groups (clubs, districts, jurisdictions). Of course, the details of the solution depend upon the specific features of the problem, among which the reasons why agents desire to act collectively within jurisdictions, but irrespective of those aspects, the description of an equilibrium (or stable) partition amounts to the three following items: (1) equilibrium number of jurisdictions; (2) equilibrium sizes of the jurisdictions; and (3) equilibrium composition of the jurisdictions.

The first question has attracted most of the attention. At the core of most models of jurisdiction formation is some type of increasing returns to scale which is contrasted with the heterogeneity of agent's characteristics and tastes. The increasing returns to scale tends to favor the creation of large groups, whereas the group heterogeneity may tip the scale in favor of smaller groups. The equilibrium number of jurisdictions results from the trade-off between these two forces.

The second question has been much less explored from a theoretical perspective. Given the equilibrium number of $M$ jurisdictions, consider the $M$-dimensional vector, the coordinates of which represent the mass of individuals in each jurisdiction. Then, we can compute various indices that offer alternative perspectives on the degree of heterogeneity exhibited by the vector of jurisdictional sizes that describes the distribution of individuals across jurisdictions. There is an important empirical literature, such as the examination of distribution of the population across different units (cities, counties, metropolitan areas, regions, countries), which focuses on the characteristics of the above vector. The theoretical analysis of the economic mechanisms underlying population mobility offers a wide range of models contrasting forces towards agglomeration and the different costs resulting from

\footnotetext{
${ }^{1}$ The literature on the formation of jurisdictions is vast and spans many different areas including among others game theory, general equilibrium and local public finance (Westhoff (1977), Wooders (1978, 1980), Guesnerie and Oddou (1981, 1988), Greenberg and Weber (1986, 1993), Demange(1994)).
} 
being in a very populated unit. ${ }^{2}$ There are two robust empirical regularities in the urban setting. ${ }^{3}$ One is that the largest cities satisfy Zipf's law asserting that their sizes are inversely proportional to their rank, whereas the other (often called the Gibrat's law) postulates that the growth rate of city populations does not depend on the size of the city. ${ }^{4}$ In our paper, the magnitude of heterogeneity in sizes displayed by a jurisdictional structure $P$ is captured through an index $H(P)$ defined as the ratio between the largest and the smallest sizes.

The third question is equally important. Our paper considers an environment where each agent prefers a jurisdiction populated with agents whose types are similar to her own. It is more difficult to reach a compromise when the population of the jurisdiction is heterogeneous: if the willingness to pay for public goods (vertical aspects) as well as preferences over public expenditures (horizontal aspects) are similar within the jurisdiction, it is easier to facilitate the derivation of a commonlyshared public policy. To make it short, intra-jurisdictional heterogeneity is costly. This class of environments should be contrasted with those in which agents prefer instead to be with agents with a different type. ${ }^{5}$ In our paper, like in many others, we assume further that the type consists of a one dimensional parameter. Then, for a given size of jurisdiction, homogeneity is maximal when the jurisdiction is an interval. In local public finance, this qualitative feature describing the composition by types of the group is often referred to as stratification while in game theory it is called consecutiveness.

In this paper we consider a model of provision of public goods which are differentiated along a horizontal axis, where public goods are differentiated on the basis of a single parameter the preferences over which intrinsically differ across agents. We will often refer to this parameter as location, but it could be examined in different settings. If the public good is a school district as in Alesina, Baqir and Hoxby (2004), this parameter represents a degree of bilingual education or number of courses

\footnotetext{
${ }^{2}$ Eeckhout (2004) proposes such a model to explain the distribution of a homogeneous population across cities. On one hand, large cities are preferred by firms and workers because of the presence of various local externalities while on the other hand, workers are exposed to higher property prices and commuting costs.

${ }^{3}$ See Rose (2005) for similar empirical observations with respect to countries rather than cities.

${ }^{4}$ The two laws seem to contradict each other. Several resolutions of this puzzle have been proposed (see e.g. Gabaix (1999) and Eeckhout (2004)).

${ }^{5}$ See for instance the model of agglomeration proposed and studied by Ellison and Fudenberg (2003) where agents prefer to be in a market with fewer agents of their own type.
} 
in black history. The type of an agent is assumed to be real number representing her ideal choice of public good: any departure from the favorite choice incurs a cost which will be assumed to be linear with respect to the distance to her ideal point. Following Alesina and Spolaore (1997), we will further assume that heterogeneity in the population is described by the uniform distribution over the unit interval. Within that model, we answer the three questions posed above for two equilibrium concepts, Nash stability and local Nash stability. We also evaluate the degree of heterogeneity of jurisdictional sizes in stable partitions by employing two indices, the size ratio of the largest and the smallest jurisdiction in a partition, and the variance of the distribution of jurisdictional sizes.

We offer a full characterization of the set of Nash stable jurisdictional structures. We demonstrate that intra-jurisdictional heterogeneity is minimal as all jurisdictions are intervals. We show all that there are always Nash stable homogeneous jurisdictional structures that consist of jurisdictions of equal size. However, there are also Nash stable structures with heterogenous jurisdictional sizes. We also show that there is no upper bound of the size ratio the large and the small jurisdiction is not bounded from above. We then proceed to a similar investigation in the case of local Nash stability (which is more demanding concept that mere Nash stability) that is tested against deviations by intervals of arbitrary small positive measure. We first show that the sizes of all the jurisdictions in a locally Nash stable partition are bounded from below by a threshold that depends on the cost of the public good. The main result asserts that a locally Nash stable jurisdictional structure exists if and only if the cost of the public good is neither too high nor too low. The jurisdictions could be of two different sizes, large and small, and the maximal heterogeneity exhibited by a jurisdictional structure is bounded from above. The restriction on the maximal possible variance is also evaluated.

\section{Related Literature}

The framework of this paper is that of Alesina and Spolaore (1997) (AS - henceforth) and we assume that the distribution of types is uniform on the unit interval. AS examine the implications of several alternative notions of stability but demonstrate that only homogeneous jurisdictional structures pass the tests. Since there is no jurisdictional size heterogeneity in their framework, AS only examine the impact of the cost of the public good on the number of jurisdictions. Le Breton and We- 
ber (2003), Haimanko, Le Breton and Weber (2004, 2005) have considered non-uniform population distributions and studied the consequences of polarization on both the efficient and the equilibrium jurisdictional structures. In this paper, we maintain the assumption of uniformity to focus on the role of increasing returns captured by the cost of the public good and contrast our results with AS. Bogomolnaia et al. (2006) explore some of the questions addressed in this paper for arbitrary discrete probability distributions. In particular, it is shown there that the existence of stable jurisdiction structures is not guaranteed.

An important contribution of Alesina, Baqir and Hoxby (2004) examines the case where the initial territory to be divided is a county and the jurisdictions are either the school districts, the school attendance areas, the municipalities or the special districts within the county. The theory presented considers differences between school districts as being horizontal. They examine the impact of the different forms of heterogeneity on the resulting number of school districts and find strong evidence of the impact of racial and income heterogeneity but little evidence that jurisdictions are shaped by ethnic or religious heterogeneity. However, Alesina, Baqir and Hoxby focus only on the number of jurisdictions, so that an empirical attempt to investigate characteristics of existing jurisdiction structures together with a test of theories intended to explain the observed outcomes, is left for the future research.

The paper is organized as follows. In the next section we present the model. Sections 3 and 4 are devoted to the examination of the concepts of Nash stable and Locally Nash stable partitions, respectively. In Section 5 we offere some concluding remarks. The proofs of all results are relegated to the Appendix.

\section{The Model}

The society, represented by a continuum of individuals uniformly distributed over the unit interval $I=[0,1]$, faces a problem of location and financing of public facilities (e.g., libraries), and a partition of individuals into jurisdictions, each assigned to one public facility. The facilities can be located anywhere on $I$ and the cost of each facility is given by a positive number $g$, independent of the 
facility location and the number of individuals assigned to it.

Every measurable subset $S$ of $I$ is a potential candidate to form a jurisdiction and we denote by $M(S)$ the set of its median locations:

$$
M(S)=\left\{p \in I: \lambda(\{t \in S: t \leq p\})=\lambda(\{t \in S: t \geq p\})=\frac{1}{2} \lambda(S)\right\}
$$

where $\lambda(\cdot)$ is the Lebesgue measure on $I$.

Each individual incurs a linear transportation cost from her location to that of public facility, that is, the transportation cost of individual located at $t$ to the facility located at $p$ is given by $d(t, p)=|t-p|$. It would be also useful to introduce the aggregate transportation cost of the set $S$ given by:

$$
D(S)=\min _{p \in I} \int_{S} d(t, p) d t
$$

It is easy to see that the minimum in (2) is attained at $p \in M(S)$. Thus, the efficiency principle requires that whenever jurisdiction $S$ forms, it selects a public facility location $p$ from the set $M(S)$. Since the set $M(S)$ is an interval and may contain more than one element, we assume that in such circumstances the jurisdiction selects the middle point $m(S)$ from the set $M(S)$. (If $M(S)$ is itself is a single point, then $m(S)$ obviously coincides with $M(S)$ ).

We assume that all formed (and potential) jurisdictions shared the cost of the facility $g$ equally among its members. Thus, the total cost incurred by an individual $t$ in jurisdiction $S$, which consists of her transportation cost and monetary contribution towards public facility, is equal to:

$$
c(t, S) \equiv|t-m(S)|+\frac{g}{\lambda(S)}
$$

For a fixed $S$, it is straightforward to check that this function is piecewise linear (and therefore continuous) as a function of $t$. Let us now introduce the concept of $n$-jurisdiction structure for an arbitrary positive integer $n$ :

Definition 2.1: A $n$-jurisdiction structure or partition $^{6}$ is a family $P=\left(S_{i}\right)_{1 \leq i \leq n}$ of $n$ measurable sets of positive measure with pairwise disjoint interior whose union is equal to the entire set

\footnotetext{
${ }^{6}$ In cooperative game theory this notion is called a coalition structure (Aumann and Dreze (1974), Greenberg (1994)).
} 
$I .^{7}$ An $n$-jurisdiction structure $P$ is stratified or consecutive if every set $S_{i} \in P$ is an interval.

A stratified $n$-jurisdiction structure is determined by $n-1$ points $0<x_{1}<\ldots<x_{n-1}<1$ : $S_{i}=\left[x_{i-1}, x_{i}\right]$ for $i=1, \ldots, n$, where, by definition, $x_{0}=0$, and $x_{n}=1$.

We now turn to main issue of this paper, the degree of a size heterogeneity displayed by a jurisdiction structure. We introduce two notions. The first is the heterogeneity gap defined as the ratio between the size of the largest and the smallest jurisdiction in $P$ and the second is the variance of jurisdiction sizes in $P$ :

Definition 2.2: Let $P$ be an $n$-jurisdiction structure. Let $\bar{s}(P)$ and $\underline{s}(P)$ denote the sizes of the largest and smallest jurisdictions in $P$, respectively. Then the heterogeneity gap, $H(P)$, is defined by their ratio:

$$
H(P)=\frac{\bar{s}(P)}{\underline{s}(P)} .
$$

The size variance $V(P)$ of an $n$-jurisdiction structure $P$ is defined as:

$$
V(P)=\sum_{S \in P}\left(\lambda(S)-\frac{1}{n}\right)^{2} .
$$

The $n$-jurisdiction structure which consists of jurisdictions of equal size $\frac{1}{n}$ (for which $H(P)=1$ and $V(P)=0$ ) will be called homogeneous. All other partitions will be called heterogenous.

In the next section we examine the notion of Nash stable structures.

\section{Nash Stability}

This section is devoted to the examination of the implications of Nash stability and the characterization of Nash stable jurisdiction structures. Note that in a model with a continuum of agents, the action of a single individual has no impact on the aggregate outcome:

\footnotetext{
${ }^{7}$ If one allows for null-set jurisdictions, then their members would incur infinitely high costs; and such a jurisdiction could be merged with any other jurisdiction of positive measure, without affecting the cost of the members of the latter. The pairwise disjoint interior simply means that some jurisdictions may have common border points, which would not affect our results.
} 
Definition 3.1: A jurisdiction structure $P=\left\{S_{1}, \ldots, S_{n}\right\}$ is Nash stable if $c\left(t, S^{t}\right) \leq c\left(t, S_{i}\right)$ for all $t \in I$ and for every $S_{i} \in P$, where $S^{t}$ denotes the jurisdiction in $P$ that contains $t .{ }^{8}$ For a given project cost $g$ and $n>0$, we denote by $\mathcal{N}(g, n)$ the set of all Nash stable $n$-partitions.

Nash stability simply requires that the no individual would desire to move from her current jurisdiction to another one.

Obviously, the set $I$ itself is trivially a Nash stable 1-partition. The following proposition describes some preliminary properties of Nash stable jurisdiction structures.

Proposition 3.2: (i) Every Nash stable jurisdiction structure $P$ is stratified.

(ii) A stratified $n$-jurisdiction structure $P=\left(x_{1}, \ldots, x_{n-1}\right)$ is Nash stable if and only if the function $c\left(t, S^{t}\right)$ is continuous in $t$ over the set $I$.

Since every Nash stable structure is stratified, the continuity requirement has to be verified only at locations of those individuals $t$, called peripheral, who are located at the border of two adjacent jurisdictions. Every peripheral individual located at one of the border points $x_{i}, i=1, \ldots, n-1$, is indifferent between the two adjacent jurisdictions ${ }^{9}\left[x_{i-1}, x_{i}\right]$ and $\left[x_{i}, x_{i+1}\right]$, i.e., $c_{x_{i}}\left(S_{i}\right)=c_{x_{i}}\left(S_{i+1}\right)$ for every $i=1, \ldots n-1$. The simple observation above leads to a formulation of Nash stability in terms of an elementary system of $n-1$ equations with $n-1$ variables which is, in fact, a recursive equation of the second order. Namely, $P=\left(x_{1}, \ldots, x_{n-1}\right)$ is Nash stable if and only if

$$
\frac{x_{i}-x_{i-1}}{2}+\frac{g}{x_{i}-x_{i-1}}=\frac{x_{i+1}-x_{i}}{2}-x_{i}+\frac{g}{x_{i+1}-x_{i}}
$$

for every $i=1, \ldots, n-1$. The last condition can be rewritten as

$$
\Psi\left(s_{i}\right)=\Psi\left(s_{i+1}\right) \text { for all } i=1, \ldots, n-1
$$

where $s_{i}=x_{i}-x_{i-1}$ and $\Psi(s)$ denotes the total cost incurred by a peripheral individual in a jurisdiction of size $s$ :

$$
\Psi(s) \equiv \frac{g}{s}+\frac{s}{2} .
$$

\footnotetext{
${ }^{8}$ Given Definition 2.1, the set $S^{t}$ may not be well-defined over a null set of individuals. We will show, however, that in stratified partitions those individuals incur the same cost in both jurisdictions they belong.

${ }^{9}$ This condition is called border indifference in Bogomolnaia et al. (2005).
} 
The function $\Psi$ is strictly convex and attains its minimum at $s^{*}=\sqrt{2 g}$, which is the optimal jurisdictional size from the point of view of the peripheral individual. Since $\lim _{s \rightarrow 0} \Psi(s)=\lim _{s \rightarrow \infty} \Psi(s)=\infty$, for any $w>\Psi\left(s^{*}\right)=s^{*}$, there are two values of $s$ that satisfy $\Psi(s)=w$. This yields a useful observation:

Proposition 3.3: Every Nash stable jurisdiction structure is either homogeneous or consists of jurisdictions of two different sizes which yield the same value of $\Psi$.

We have the following results concerning Nash stable partitions:

Proposition 3.4: Let $n>1$ be an integer. Then

(i) The homogeneous $n$-jurisdictional structure is Nash stable.

Denote $g_{n}=\frac{1}{8(n-1)}$ for every $n$.

(ii) A Nash stable heterogenous 2-jurisdiction structure exists if and only if $g<g_{2}$.

(iii) For $n \geq 3$, a Nash stable heterogenous $n$-jurisdiction structure exists if and only if $g \leq g_{n}$.

Regarding three questions formulated in the introduction, Nash stability does not impose any bounds on the number of jurisdictions in a homogenous partition and places an upper bound for heterogenous partitions. There could be an inter-jurisdictional heterogeneity when a stable structure may contain jurisdictions of two possible sizes. Finally, Nash stability implies stratification, and, thus, intrajurisdictional homogeneity.

Now let us evaluate the heterogeneity gap and the variance for Nash stable partitions. For any $n>0$ and cost of the project $g>0$, define $H^{\mathcal{N}}(n, g)$ and $V^{\mathcal{N}}(n, g)$ as:

$$
H^{\mathcal{N}}(n, g)=\max _{P \in \mathcal{N}(g, n)} H(P) \quad \text { and } \quad V^{\mathcal{N}}(n, g)=\max _{P \in \mathcal{N}(g, n)} V(P)
$$

If $\mathcal{N}(g, n)=\emptyset$, then we define $H^{\mathcal{N}}(n, g)=V^{\mathcal{N}}(n, g)=-\infty$. We have

Proposition 3.5: For every integer $n>0$ we have

(i) $\sup _{g>0} H^{\mathcal{N}}(g, n)=\infty$ and (ii) $\sup _{g>0} V^{\mathcal{N}}(g, n)=1-\frac{1}{n}$.

This proposition shows that Nash stability, in fact, does not have an impact on heterogeneity gap, and there could be Nash stable structures that contain jurisdictions with sharply distinct sizes. 


\section{Local Nash stability}

In this section, we examine the implications of a more demanding concept of locally Nash stable partition which has been introduced by Jehiel and Scotchmer $(1997,2001)$ in a somewhat different framework. This notion aims to test the stability of a given jurisdiction structure against deviations by coalitions of positive but arbitrarily small size. Indeed, if an individual finds it (strictly) beneficial to migrate, then a small neighborhood of that individual may contemplate the same migrational choice. If such a group move takes place, then it affects the outcome of the jurisdiction absorbing the migrants but also the situation in jurisdictions they left behind.

While coalitional deviations are often questioned on the basis of credibility, coordination and communication, it is important to point out that if a deviating group has to be "very small", this would eliminate most of those arguments. Formally,

Definition 4.1: An $n$-jurisdiction structure $P=\left\{S_{1}, \ldots, S_{n}\right\}$ is said to be locally Nash stable if there exists $\varepsilon>0$ such that there is no interval $T$, whose length $|T| \leq \varepsilon$, and a jurisdiction $S \in P$ such that $c(t, S \cup T)<c\left(t, S^{t}\right)$ for all $t \in T$.

For every $g>0$ and $n>0, \mathcal{L}(g, n)$ denotes the set of all locally Nash stable $n$-jurisdiction structures.

Note that the notion of a local Nash stability is weaker than stability under free mobility in Bogomolnaia et al. (2005), where there is no restriction on the size of a deviating group.

While the formal proof is provided in the Appendix, it is evident from the above discussion that local Nash stability is indeed a refinement of Nash stability:

Corollary 4.2: Every locally Nash stable jurisdiction structure is Nash stable.

Proposition 3.2(i) and Corollary 4.2 imply that any locally Nash stable jurisdiction structure is stratified. We can now exploit that property to identify small intervals which are threats to the stability of a jurisdiction structure. It turns out that jurisdiction structures with too many jurisdictions or, equivalently, whose jurisdictions are too small, are likely to be unstable. Indeed, a 
small enlargement of the jurisdiction leads to a sharp decrease of the tax paid by the residents while the transportation cost remains almost unchanged.

Proposition 4.3: Let $g>0$ and $P$ be a Nash stable $n$-jurisdiction structure with $n>1$. Then $P$ is locally Nash stable if and only if $\lambda(S) \geq \hat{g}$ for every jurisdiction $S \in P$, where $\hat{g}=\sqrt{\frac{2 g}{3}}$. Note that Proposition 4.3 implies a homogeneous $n$-jurisdiction structure is locally stable if and only if $n \leq 1 / \hat{g}$.

We can now determine the range of the project costs $g$ for which there exist locally Nash stable jurisdiction structures:

Proposition 4.4: Let $n>1$ and $g>0$. There exist two values, $g(n)<\bar{g}(n)$, such that

(i) When $n=2$, a heterogeneous locally Nash stable $n$-jurisdiction structure exists if and only if $g \in\left[\underline{g}(n), g_{n}\right)$. (Recall that $g_{n}=\frac{1}{8(n-1)}$ as we defined above).

(ii) When $n=3$, a heterogeneous locally Nash stable $n$-jurisdiction structure exists if and only if $g \in\left[\underline{g}(n), g_{n}\right] \backslash\left\{\frac{1}{2 n^{2}}\right\}$.

(iii) When $n=4,6$, a heterogeneous locally Nash stable $n$-jurisdiction structure exists if and only if $g \in[\underline{g}(n), \bar{g}(n)] \backslash\left\{\frac{1}{2 n^{2}}\right\}$.

(iv) When $n \neq 2,3,4,6$, a heterogeneous locally Nash stable $n$-jurisdiction structure exists if and only if $g \in[g(n), \bar{g}(n)]$.

The existence of the bound for homogenous partitions follows from Proposition 4.3, which also provides an upper bound on the project cost $g$ for heterogenous partitions. The argument for the existence of a lower bound is more intricate. For small values of $g$ a heterogeneous jurisdiction structure contains large jurisdictions whose size is substantially larger than the efficient size $s^{*}=\sqrt{2 g}$. Thus, the peripheral individuals in these jurisdictions incur a significant transportation cost. This, in turn, implies that peripheral individuals in small jurisdictions must share a substantial tax burden imposed by the cost of public facility. Since, by assumption, $g$ is very small, this implies that the size of these small jurisdictions is "very small", too, which may contradict Proposition 4.3. This reasoning 
suggests that in any heterogeneous jurisdiction structures, the size of small and large jurisdictions should not diverge too much or, stated differently, the heterogeneity gap should not exceed some threshold.

As in the preceding section, for any $n>0$ and $g>0$, define $H^{\mathcal{L}}(n, g)$ and $V^{\mathcal{L}}(n, g)$ as:

$$
H^{\mathcal{L}}(n, g)=\max _{P \in \mathcal{L}(g, n)} H(P) \quad \text { and } \quad V^{\mathcal{L}}(n, g)=\max _{P \in \mathcal{L}(g, n)} V(P)
$$

If $\mathcal{L}(g, n)=\emptyset$, then, as before, both $H^{\mathcal{L}}(n, g)$ and $V^{\mathcal{L}}(n, g)$ are equal to $-\infty$. We have the following result:

Proposition 4.5: (i) Let $n>1$. Then

(i) $\max _{g>0} H^{\mathcal{L}}(g, n)=3$ and $(i i) \sup _{g>0} V^{\mathcal{L}}(g, n) \leq \frac{1}{3 n}$. (The equality is strict for every $n$ of the form $n=4 m$, whereas the inequality is very tight for other values of $n$.)

We can now sort out the limitations on the set of jurisdiction structures imposed by local Nash stability. First, the number of jurisdictions is not unrestricted anymore: for a given $g$ there is an upper bound on the number of jurisdictions in the partition, or, alternatively, for a given $n, g$ has to be sufficiently small. Obviously the constraints on locally Nash stable heterogeneous jurisdiction structures are tighter than in the Nash stability case: there is a lower bound on $g$ and the upper bound on $g$ is smaller than that for Nash stability. Finally, as a subset of the Nash stable jurisdiction structures, the locally Nash stable partitions do not display any intra-jurisdictional heterogeneity.

It is important to compare the notion of local stability employed in this paper with the $A$ stability of Alesina and Spolaore (1997). To be precise, a stratified jurisdiction structure $P=$ $\left(x_{1}, \ldots, x_{n-1}\right)$ is A-stable if for all $\varepsilon>0, i=1, \ldots, n-1$ and $\mu \leq \varepsilon$, there exists $t \in\left[x_{i}, x_{i}+\mu\right]$ such that $c\left(t,\left(S_{i-1} \cup\left[x_{i}, x_{i}+\mu\right]\right) \geq c\left(t,\left[x_{i}+\mu, x_{i+1}\right]\right)\right.$. In contrast, a stratified jurisdiction structure $P=\left(x_{1}, \ldots, x_{n-1}\right)$ is locally Nash stable if for all $\varepsilon>0, i=1, \ldots, n-1$ and $\mu \leq \varepsilon$, there exists $t \in\left[x_{i}, x_{i+1}+\mu\right]$ such that $c\left(t,\left(S_{i} \cup\left[x_{i}, x_{i}+\mu\right]\right) \geq c\left(t, S_{i+1}\right)\right.$. Both definitions assert that a structure is locally stable under the condition that whenever a small group $S$ contemplates a migration to an adjacent jurisdiction, there is a member of $S$ who would prefer to stay in the original jurisdiction. 
The two definitions, however, differ in the evaluation of the benefits attached to the option of staying in the "home" jurisdiction. Under local Nash stability the home jurisdiction remains untouched if at least of one of potential migrants declines the proposed move, whereas under A-stability the migration move always takes place and all considerations are of ex post nature. Thus, the local Nash stability is a game theoretical concept that describes the robustness of the jurisdiction structure against group deviations of arbitrarily small size. It is in the spirit of strong Nash equilibrium (Aumann (1959)), where no group deviation takes place as soon as there is a member of the group who prefers the pre-deviation situation to the post-deviation one. The concept of A-stability is more of a mechanical nature that describes the response of a jurisdiction structure to a local exogenous shock. The implications of the two concepts are quite different. In particular, a straightforward calculation shows that A-stability rules out the size heterogeneity, whereas the local Nash stability does not.

\section{$5 \quad$ Synthesis and Concluding Remarks}

In this section we wish suggest several avenues for further research. We have shown that our stability concepts allow for heterogeneous jurisdiction structures. The heterogeneity, however, is limited to two different sizes. This conclusion is based on the assumption of uniform density, in absence of which one could generate more sophisticated patterns of jurisdictions'sizes. Note that the stability conditions for stratified structures can be expressed through the recursion mechanism that starts at points $\theta_{0}=0$ and $\theta_{1}$ and proceeds to generate other border points of the partition. To make it more precise, present the jurisdictional structure as a sequence:

$$
\left[\theta_{0}, \theta_{1}\right],\left[\theta_{1}, \theta_{2}\right], \ldots,\left[\theta_{n-1}, \theta^{0}\right]
$$

where $\theta^{0}=1$. Then

$$
\theta_{i}-m\left(\theta_{i-1}, \theta_{i}\right)+\frac{g}{F\left(\theta_{i}\right)-F\left(\theta_{i-1}\right)}=m\left(\theta_{i+1}, \theta_{i}\right)-\theta_{i}+\frac{g}{F\left(\theta_{i+1}\right)-F\left(\theta_{i}\right)},
$$

where $F$ denotes the cumulative function of the population distributed over the interval $[0,1]$, and $m\left(\theta, \theta^{\prime}\right)$ is the median of the interval $\left[\theta, \theta^{\prime}\right]$. This leads to a second order "dynamical" system 
$\theta_{i+1}=h\left(\theta_{i}, \theta_{i-1}\right)$, where $h$ is a multi-valued function derived from solving (8). In the case of the uniform distribution, we obtain

$$
\theta_{i+1}=2 \theta_{i}-\theta_{i-1}, \text { or } \theta_{i+1}=\theta_{i}+\frac{2 g}{\theta_{i}-\theta_{i-1}}
$$

In fact, in that case the "dynamical" system is equivalent to a simpler one that links the sizes of two adjacent jurisdictions by either $s_{i+1}=s_{i}$ or $s_{i+1}=2 g / s_{i}$.

Clearly, any other population density function will generate a more sophisticated recursive equation and richer patterns of heterogeneity in sizes. Along that line of research, the closest contributions are due to Jehiel and Scotchmer in a setting where the public goods are differentiated along a single vertical dimension (say, quantity) and where the type of an individual is her willingness to pay distributed uniformly over an interval $\left[\theta_{0}, \theta^{0}\right]$, where $\theta_{0}>0$. It is shown there that there is a unique locally Nash stable jurisdiction structure. The constructive proof is based on explicit computation of the second order "dynamical" system $h$, where $\theta_{i+1} / \theta_{i}=h\left(\theta_{i} / \theta_{i-1}\right)$.

It is interesting to note that the equilibrium jurisdictional structure in this setting displays strong size heterogeneity, where the rightmost jurisdiction is the largest one and contains at least half of the population. A repeated application of that argument and a more detailed estimation lead to the establishment of bounds on the heterogeneity gap, and it certainly could be the case that this approach could lead to promising results in our framework.

The two stability concepts we examine in this paper reflect the paradigm of free entry. It is therefore natural to examine what would be the predictions of stability concepts incorporating barriers to entry. In the case of local Nash stability, the arrival of a small group of migrants, say, from the right side of the jurisdiction, is welcomed by most of the residents on the right side of the median, as the migration brings them a double dividend: the cost is shared among a larger group and the location of the public facility is shifted in their direction. Others may be disadvantaged by the migration wave and could oppose it if possible. The definition of stability may also call for a precise definition of the feasibility conditions under which a profitable move can be effective. ${ }^{10}$ One such concept is the

\footnotetext{
${ }^{10}$ See Bogomolnaia and Jackson (2002) for general results.
} 
B-stability of Alesina and Spolaore (1997). In a companion paper, Bogomolnaia et al. (2005) provide a characterization of the set of stable jurisdictions where no new group can be created under the requirement that its members would unanimously prefer a new jurisdiction rather over their current ones. The insights gained there suggest additional directions of the future research.

\section{Appendix}

Proof of Proposition 3.2: (i) Suppose, in negation, that there exists a non-stratified Nash stable jurisdiction structure $P$. This implies that there exist $S, T \in P$, and $s_{1}, s_{2} \in S, s^{*} \in T$ such that $s_{1}<s^{*}<s_{2}$. Let, without loss of generality, $m(S)<m(T)$. Consider the following cases.

Case 1. $c(t, S) \geq c(t, T)$ for all $t \in I$. If the inequality is always strict, every individual in $S$ would migrate to $T$. If not, we must have $c(m(S), S)=c(m(S), T)$, and, therefore, $c(t, S)>c(t, T)$ for all $t>m(S)$. Then every individual in $S$ located to the right of $m(S)$ would rather migrate to $T$, a contradiction to the Nash stability of $P$.

Case 2. $c(t, S) \leq c(t, T)$ for all $t \in I$. It can be examined in the same manner as Case 1 .

Case 3. The two sets, $\{t \in I \mid c(t, S)<c(t, T)\}$ and $\{t \in I \mid c(t, S)>c(t, T)\}$, have a positive measure. Then the difference $\delta(t)=c(t, S)-c(t, T)$ is nondecreasing in $t$ on $I$. Moreover, it satisfies $\delta(m(S))<0$ and $\delta(m(T))>0$ and strictly increases on $[m(S), m(T)]$.

Since $s^{*} \in T, c\left(s^{*}, S\right) \geq c\left(s^{*}, T\right)$ and $\delta\left(s^{*}\right) \geq 0$. Thus, $s^{*}>m(S)$, and $\delta\left(s_{2}\right)>0$, yielding $c\left(s_{2}, S\right)>c\left(s_{2}, T\right)$. That is, $s_{2}$ would rather move to $T$, again contradicting our assumption of Nash stability of $P$.

(ii) It is sufficient to prove, for a stratified partition $P=\left(x_{1}, \ldots, x_{n}\right)$, the equivalence between Nash stability and border indifference, which amounts to the equalities $c\left(x_{i}, S_{i}\right)=c\left(x_{i}, S_{i+1}\right)$ for all $i=1, \ldots, n-1$.

Let $P$ be a Nash stable stratified jurisdiction structure, and assume that there exists $i$ such that, without loss of generality, $c\left(x_{i}, S_{i}\right)>c\left(x_{i}, S_{i+1}\right)$. Since the functions $c\left(\cdot, S_{i}\right)$ and $c\left(\cdot, S_{i+1}\right)$ are continuous, there exists an interval $T \equiv\left[x_{i}-\mu, x_{i}\right] \subset S_{i}$ such that $c\left(t, S_{i}\right)>c\left(t, S_{i+1}\right)$ for all $t \in T$, a contradiction to the Nash stability of $P$. 
Conversely, assume that $c\left(x_{i}, S_{i}\right)=c\left(x_{i}, S_{i+1}\right)$ for all $i=1, \ldots, n-1$, or, equivalently, the function $c\left(t, S^{t}\right)$ is continuous in $t$. For any $S \subset I$, denote by

$$
\Delta_{S}(t) \equiv c(t, S)-c\left(t, S^{t}\right)
$$

the difference between the payoff that individual $t$ receives while staying at his home jurisdiction, and that she would receive from migrating to jurisdiction $S$. We claim that this function is continuous (actually, piecewise linear) and (weakly) single-dipped, with a minimum at $m(S)$.

Continuity follows directly from our assumption. To prove single-dippedness, it is enough to show that the function $\Delta_{S}(t)$ is non-increasing for $t \leq m(S)$ (the case $t \geq m(S)$ is treated in a similar manner).

By continuity of $c\left(t, S^{t}\right)$, it suffice to consider each jurisdiction separately. Consider jurisdiction $S_{i}$ such that $m\left(S_{i}\right)<m(S)$. Put $R_{i}=\frac{g}{\lambda[S]}-\frac{g}{\lambda\left[S_{i}\right]}$ and let $t \in S_{i}$. Then

$$
\begin{gathered}
\Delta_{S}(t)=|t-m(S)|-\left|t-m\left(S_{i}\right)\right|+R_{i}= \\
\begin{cases}m(S)-m\left(S_{i}\right)+R_{i}, & t \leq m\left(S_{i}\right) ; \\
m(S)+m\left(S_{i}\right)+R_{i}-2 t, & t \geq m\left(S_{i}\right) .\end{cases}
\end{gathered}
$$

Since $\Delta_{S}(t)$ is single-dipped with a minimum at $m(S)$, and obviously $\Delta_{S_{i}}\left(m\left(S_{i}\right)\right)=0$ for all $i=1, \ldots, n$, we conclude that $c\left(t, S^{t}\right) \leq c\left(t, S_{i}\right)$ for all $t \in I$ and all $i=1, \ldots, n$, i.e. $P$ is Nash stable.

Proof of Proposition 3.4: (i) follows directly from (6) as all the equations are satisfied when $s_{i}=\frac{1}{n}$ for all $i=1, \ldots, n$.

(ii) Let $P$ be a Nash stable heterogenous $n$-jurisdiction structure. Then, there are exactly two different values in the bundle $\left(s_{1}, \ldots, s_{n}\right)$ (at least two, due to heterogeneity, and no more than two, since equation $\Psi(s)=w$ has at most two solutions). Let $w$ denotes the common value of $\Psi\left(s_{k}\right)$. The two different sizes, $s$ and $s^{\prime}$ are the roots of the quadratic equation

$$
\Psi(s)=\frac{s}{2}+\frac{g}{s}=w
$$

where $s=w-\sqrt{w^{2}-2 g}$ and $s^{\prime}=w+\sqrt{w^{2}-2 g}$ as long as $w \geq \sqrt{2 g}$. Since there are no real roots for $w<\sqrt{2 g}$ and a single root for $w=\sqrt{2 g}$, in any heterogeneous $n$-jurisdiction structure we should 
have $w>\sqrt{2 g}$. Let $k$ be the number of small jurisdictions of size $s$ and $l$ be the number of large jurisdictions of size $s^{\prime}$, where $k+l=n$. Since $k s+l s^{\prime}=1$, we obtain:

$$
l\left(w+\sqrt{w^{2}-2 g}\right)+k\left(w-\sqrt{w^{2}-2 g}\right)=n w+(l-k) \sqrt{w^{2}-2 g}=1 .
$$

If $n=2$, we have $l=k=1$ and (12) turns into $2 w=1$. Thus, a Nash stable 2-jurisdiction structure exists if and only if $1=2 w>2 \sqrt{2 g}$ or $g<g_{2}$.

Consider now the case where $n>2$. As $l>0$ in any heterogeneous structure, we must have $k<n$. Since both $k$ and $l$ are integers, $l-k \geq 2-n$ and

$$
1=n w+(l-k) \sqrt{w^{2}-2 g} \geq n w-(n-2) \sqrt{w^{2}-2 g} \geq \min _{w>\sqrt{2 g}}\left\{n w-(n-2) \sqrt{w^{2}-2 g}\right\}
$$

The expression $n w-(n-2) \sqrt{w^{2}-2 g}$ attains its minimum when

$$
w=\frac{n \sqrt{2 g}}{\sqrt{n^{2}-(n-2)^{2}}}>\sqrt{2 g} .
$$

The minimal value is equal to $\sqrt{\left[n^{2}-(n-2)^{2}\right] \cdot 2 g}$. Hence, if a heterogeneous Nash stable $n$ jurisdiction exists, we must have $1 \geq \sqrt{\left[n^{2}-(n-2)^{2}\right] \cdot 2 g}$ or $g<g_{n}$. This completes the "only if" part of the proof. The proof of the "if" part utilizes the property stated in the following auxiliary proposition, the proof of which is obtained by simple calculus:

Lemma A.1: The expression

$$
n w-(k-l) \sqrt{w^{2}-2 g}
$$

increases monotonically in $w$ for $w \in[\sqrt{2 g},+\infty)$, when $k \leq l$. It is single-dipped with the minimum at

$$
w=\frac{n}{\sqrt{n^{2}-(k-l)^{2}}} \sqrt{2 g}
$$

when $k>l$. This expression is unbounded from above when $w$ approaches infinity.

If $g=g_{n}$, then the $(n-1,1)$ jurisdiction structure is Nash stable since $w=\frac{n \sqrt{2 g}}{\sqrt{n^{2}-(n-2)^{2}}}>\sqrt{2 g}$. If $g<g_{n}$, the collection of $n-1$ small jurisdictions and one large jurisdiction corresponding to that value of $w$ is not an $n$-jurisdiction structure any longer as we have $(n-1) s+\bar{s}<1$. 
However, the expression $n w-(n-2) \sqrt{w^{2}-2 g}=1$ is continuous and due to Lemma A.1, unbounded from above, hence, there exists $w$ such that $n w-(n-2) \sqrt{w^{2}-2 g}=1$. By construction, the corresponding heterogeneous $(n-1,1)$ jurisdiction structure is Nash stable.

Proof of Proposition 3.5: (i) From proposition A1, for any small $g$ we can always find a heterogeneous Nash stable $(k, l)$ jurisdiction structure with $w \in\left[\frac{n}{\sqrt{n^{2}-(k-l)^{2}}} \sqrt{2 g},+\infty\right)$. Let $k=n-1$ and $l=1$. As $g$ converges to 0 , the size of small jurisdictions tends to 0 as well since it is smaller than $\sqrt{2 g}$. Hence, the size of the unique large jurisdiction converges to 1 and therefore, $\lim _{g \rightarrow 0} \bar{s} / \underline{s}=+\infty$.

(ii) A simple rearrangement of $V(P)$ yields:

$$
V(P)=\sum_{i=1}^{n} s_{i}^{2}-\frac{1}{n}
$$

Since for all $i=1, \ldots, n s_{i} \leq 1$, we obtain $s_{i}^{2} \leq s_{i}$ and therefore $\sum_{i=1}^{n} s_{i}^{2} \leq \sum_{i=1}^{n} s_{i}=1$. This implies that for any stratified jurisdiction structure $P=\left(s_{1}, \ldots, s_{n}\right): V(P) \leq 1-\frac{1}{n}$. Consider a Nash stable $(n-1,1)$ jurisdiction structure and let $g \rightarrow 0$. Since as before $s$ converges to 0 and $\bar{s}$ converges to 1 , $\sum_{i=1}^{n} s_{i}^{2} \rightarrow 1$ and $V(P) \rightarrow 1-\frac{1}{n}$.

Proof of Corollary 4.2: Let $P$ be a locally Nash stable jurisdiction structure and assume on the contrary that $P$ is not Nash stable i.e, there exists $i$ such that, without loss of generality, $c\left(x_{i}, S_{i}\right)>c\left(x_{i}, S_{i+1}\right)$. The continuity of the functions $c\left(t, S_{i}\right)$ and $c\left(t, S_{i+1}\right)$ implies that there exists $T=\left[x_{i}-\mu, x_{i}\right] \subset S_{i}$ such that $c\left(t, S_{i}\right)>c\left(t, S_{i+1}\right)$ for all $t \in T$. We can choose $\mu$ small enough so that $m\left(T \cup S_{i+1}\right) \geq x_{i}$. Since $\lambda\left(S_{i+1} \cup T\right)>\lambda\left(S_{i+1}\right)$ and $x_{i}<m\left(S_{i+1} \cup T\right)<m\left(S_{i+1}\right)$, it follows that $c\left(t, S_{i+1}\right)>c\left(t, T \cup S_{i+1}\right)$, and, therefore, $c\left(t, S_{i}\right)>c\left(t, T \cup S_{i+1}\right)$ for all $t \in T$, a contradiction to $P$ being locally Nash stable.

We now use the following proposition that allows us to identify those small intervals which 
represent an actual threat to local Nash stability of jurisdiction structures. Naturally, it turns out that those intervals are located on the other side of the border with jurisdictions they consider as migration targets:

Lemma A.2: A stratified jurisdiction structure $P=\left(x_{1}, \ldots, x_{n-1}\right)$ is locally Nash stable if and only if there is an $\varepsilon>0$ such that there does not exist a jurisdiction $S_{i} \in P$ and $\mu \leq \varepsilon$ for which one of two inequalities hold:

$$
\begin{array}{r}
c\left(t, S_{i} \cup\left[x_{i}-\mu, x_{i}\right]\right)>c\left(t, S_{i-1}\right) \text { for all } t \in\left[x_{i}-\mu, x_{i}\right] \\
c\left(t, S_{i} \cup\left[x_{i+1}, x_{i+1}+\mu\right]\right)>c\left(t, S_{i+1}\right) \text { for all } t \in\left[x_{i+1}, x_{i+1}+\mu\right] .
\end{array}
$$

Proof: Consider a stratified jurisdiction structure $P$ satisfying the properties (18)-(19) stated above, and let us show that it is locally Nash stable. Let $\varepsilon>0$ be such that the properties hold and assume, without loss of generality, that $\varepsilon<\min _{i=j, \ldots, n} \lambda\left(S_{j}\right)$.

Assume that $P$ is not locally Nash stable. Then there exists an interval $J$ (not adjacent to some $\left.S_{i}\right)$ with $\lambda(J)<\varepsilon$ and a jurisdiction $S_{i} \in P$ such that $c\left(t, S^{t}\right)>c\left(t, J \cup S_{i}\right)$ for all $t \in J$. If $J \cap S_{i} \neq \emptyset$, then $\lambda\left(J \backslash S_{i}\right)<\varepsilon$ and the interval $J \backslash S_{i}$, adjacent to $S_{i}$, satisfies on of the two previous equations, thus contradicting the stated property. Consider now the case where $J \cap S_{i}=\emptyset$. Since $\lambda[J]<\varepsilon<\min _{j=1, \ldots, n} \lambda\left[S_{j}\right]<\lambda\left[S_{i}\right]$, it follows that $m\left(S_{i} \cup J\right) \notin J$.

Note now that the value of the function $\Delta_{S_{i} \cup J}(t)$, defined in (9), is negative for all $t \in J$. Then, from the properties of the function $\Delta_{S}(t)$ established in the proof of Proposition 3.2, it follows that $\Delta_{S_{i} \cup J}(t)<0$ for all $t$ between $m\left(S_{i} \cup J\right)$ and the set $J$, and, in particular, for all $t$ located between the jurisdiction $S_{i}$ and the interval $J$.

Let $J^{\prime}$ be the interval of the same size as $J$ but adjacent to $S_{i}$ on the same side of $S_{i}$ as $J$. Since $m\left(S_{i} \cup J\right)=m\left(S_{i} \cup J^{\prime}\right)$ and $\lambda\left(S_{i} \cup J\right)=\lambda\left(S_{i} \cup J^{\prime}\right)$ we conclude that $\forall t \in J^{\prime}$ the inequality $c\left(t, S^{t}\right)>c\left(t, S_{i} \cup J\right)=c\left(t, S_{i} \cup J^{\prime}\right)$ holds, a contradiction to the stated property on $P$.

Proof of Proposition 4.3: Consider a jurisdiction $S$ of a size $s$ in a Nash stable partition $P$ and 
an interval $J$ of size $\mu$ adjacent to $S$ on the right. It is straightforward to check that $\Delta_{S \cup J}\left(t^{\prime}\right) \geq 0$ for all $t^{\prime} \in J$ if and only if this inequality holds for $t$, the right endpoint of the interval $J$.

Since P is Nash stable, $c\left(t, S^{t}\right)=\Psi(s)-\mu$. Moreover, $c(t, S \cup J)=\Psi(s+\mu)$ as $t$ is the endpoint of the new interval jurisdiction $S \cup J$ of size $s+\mu$. Thus,

$$
\Delta_{S \cup J}(t)=\Psi(s+\mu)-\Psi(s)+\mu \geq 0 \Leftrightarrow \Psi(s+\mu)-\Psi(s) \geq-\mu .
$$

Since $\frac{d^{2} \Psi(s)}{d s^{2}}=\frac{2 g}{s^{3}}>0$, inequality (20) holds for all $\mu>0$ if and only if $\frac{d \Psi(s)}{d s} \geq-1$.

As $\frac{d \Psi(s)}{d s}=\frac{1}{2}-\frac{g}{s^{2}}$, we deduce that $\frac{1}{2}-\frac{g}{s^{2}} \geq-1$ if and only if $s \geq \hat{g}$.

Proof of Proposition 4.4: For $n>1$ denote

$$
\underline{g}(n)=\frac{3}{2(3 n-2)^{2}}, \text { and } \bar{g}(n)=\frac{3}{2(n+2)^{2}} .
$$

Proof of (i): For $n=2$, there exists a heterogeneous Nash stable jurisdiction structure if $g<g_{2}=\bar{g}(2)=\frac{1}{8}$. It is locally Nash stable if and only if the size of the small jurisdiction is at least $\hat{g}$. Since when $n=2, w=\frac{1}{2}$ in (11), we deduce that the partition it is locally Nash stable if and only if

$$
\frac{1}{2}-\sqrt{\frac{1}{4}-2 g} \geq \hat{g} \Leftrightarrow g \geq \frac{3}{32}=\underline{g}(2)
$$

To proceed with other cases, recall that every heterogenous locally Nash stable $n$-partition consist of jurisdictions of two sizes, $s$ and $s^{\prime}$. From (11) and Proposition 4.3 we have $s s^{\prime}=2 g$, and moreover,

$$
\hat{g} \leq s<\sqrt{2 g}<s^{\prime}
$$

As in the proof of proposition 3.4, consider a collection of $k$ jurisdictions of size $s$ and $n-k$ jurisdictions of size $s^{\prime}=\frac{2 g}{s}$. Denote by $L(k, s)$ the total size of this collection:

$$
L(k, s)=k s+\frac{2 g(n-k)}{s} .
$$

This collection turns into a heterogenous locally Nash stable $n$-partition for some $k, 1<k<n$ and $s$ satisfying (22), if $L(k, s)=1$, the total population mass of $I$. It is useful to point out that the function 
$L(k, \cdot)$ is single-dipped on the relevant interval $(0, \sqrt{2 g})$ with a minimum at $s_{m}(k)=\sqrt{(n-k) / k} \sqrt{2 g}$ if $k>n / 2$ and is monotonically decreasing if $k \leq n / 2$.

We will use the following results:

Lemma A.3: If $g>\frac{1}{2 n^{2}}$, and the set of all heterogeneous locally stable $n$-structures is nonempty, it contains a partition into $n-1$ small and one large size jurisdiction. Moreover, if $n>3$ and $g>\bar{g}(n)$ then there are no locally stable heterogeneous $n$-partitions.

Proof: Suppose that there is a heterogenous locally stable $n$-partition for $k^{\prime}<n$-1, i.e., there exists $s$ satisfying $(22)$ such that $L\left(k^{\prime}, s\right)=1$. But then $L(n-1, s)<1$. For $g>\frac{1}{2 n^{2}}$, it is easy to verify that $L(k, \sqrt{2 g})>1$ for all $1<k<n$. By continuity of the function $L$, there exists $\bar{s}, s<\bar{s}<\sqrt{2 g}$ such that $L(n-1, \bar{s})=1$.

Let $n>3$. Then the minimum $s_{m}(n-1)$ of the function $L(n-1, \cdot)$ is less than or equal to $\hat{g}$. Since $L(n-1, \sqrt{2 g})>1$, it follows that there is no heterogenous Nash locally stable $n$-structure if $L(\hat{g})>1$, or $g>\bar{g}(n)$.

Lemma A.4: If $g<\frac{1}{2 n^{2}}$, and the set of all heterogeneous locally stable $n$-structures is nonempty, it contains a partition into one small and $n-1$ large size jurisdictions. The latter occurs if and only if

$$
L(1, \hat{g}) \geq 1, \text { or } \text { org } \geq \underline{g}(n)=\frac{3}{2(3 n-2)^{2}} .
$$

Proof: Suppose that there is a heterogenous locally stable $n$-partition for $k^{\prime}>1$, i.e., there exists $s$ satisfying $(22)$ such that $L\left(k^{\prime}, s\right)=1$, hence, $L(1, s)>1$. For $g<\frac{1}{2 n^{2}}$, we have $L(k, \sqrt{2 g})<1$ for all $1<k<n$. From the continuity of $L$ we obtain the existence of $\bar{s}, s<\bar{s}<\sqrt{2 g}$ with $L(1, \bar{s})=1$. Since the function $L(1, \cdot)$ is monotonically decreasing on the interval $(0, \sqrt{2 g})$, the existence of $\bar{s}$ is guaranteed if and only if $(23)$ holds. It is easy to check that $(23)$ is equivalent to $g \geq \frac{3}{2(3 n-2)^{2}}$. Lemma A.5: If $g=\frac{1}{2 n^{2}}$, and the set of all heterogeneous Nash locally stable $n$-structures is nonempty, it contains a partition into $\frac{n}{2}+1$ small and $\frac{n}{2}-1$ large size jurisdictions for even $n$, and $\frac{n+1}{2}$ small and $\frac{n-1}{2}$ large size jurisdictions for odd $n$. Moreover, if $k>\frac{n}{2}$, then there 
exists a Nash locally stable $(k, n-k)$-partition if and only if the number of small coalitions $k$ satisfies:

$$
2 k \leq(3-\sqrt{3}) n
$$

Proof: Note that if $g=\frac{1}{2 n^{2}}$, then $L(k, \sqrt{2 g})=1$ for all $k, 1<k<n$. Let $n$ be an even number (the consideration for the odd case is very similar). Assume that there is a heterogenous locally stable $n$-partition for $k^{\prime} \neq \frac{n}{2}+1$, i.e., there exists $s$ satisfying $(22)$ such that $L\left(k^{\prime}, s\right)=1$. Then $k^{\prime}>\frac{n}{2}$, since otherwise $L\left(k^{\prime}, s\right)>1$ over the entire interval $(0, \sqrt{2 g})$.

Then we have $L\left(\frac{n}{2}+1, s\right)>L\left(k^{\prime}, s\right)=1$. Since for $\bar{s}$ close to $\sqrt{2 g}$ we have $L\left(\frac{n}{2}+1, \bar{s}\right)<1$, there exists $\tilde{s}$ such that the structure with $\frac{n}{2}+1$ small coalitions of the size $\bar{s}$ and $\frac{n}{2}-1$ large coalitions is locally Nash stable.

Now, to prove the inequality (24), consider a union of $k$ small (of size $s$ ) and $n-k$ large (of size $\left.\frac{2 g}{s}\right)$ jurisdictions such that $s=\hat{g}$. For this union, $L(k, s) \geq 1 \Leftrightarrow$ there exists a desired partition, due to single-dippedness of $L()$. But direct calculations result in $L(k, s) \geq 1 \Leftrightarrow(k+3(n-k)) \hat{g} \geq 1 \Leftrightarrow$ $2 k \leq(3-\sqrt{3}) n$.

Proof of (ii). Let $n=3$. If $g>g_{3}=\frac{1}{16}$, by Proposition 3.3 and Corollary 4.2 , then there is no heterogeneous Nash locally stable 3-jurisdiction structure, whereas, by Lemma A.3, it exists whenever $g \in\left[\underline{g}(3), \frac{1}{18}\right)$ and fails to exist if $g<\underline{g}(3)$.

Now let us consider the case where $g \in\left(\frac{1}{18}, \frac{1}{16}\right)$. Then $s_{m}(2)$, the minimum of the function $L(2, \cdot)$ is $\sqrt{g}>\hat{g}$, and $L\left(2, s_{m}(2)\right)<1$, whereas $L(2, \sqrt{2 g})>1$. Thus, there is a heterogeneous Nash stable 3 -jurisdiction structure. Finally, if $g=\frac{1}{18}$, the condition (24) in Lemma A.5 is violated for $k=2$, the only possible $k>\frac{n}{2}$, thus ruling out the existence of $s \in\left[s_{m}(2), \sqrt{2}\right)$ such that $L(2, s)=1$.

Proof of (iii). By Lemmas A.3 and A.4, there exists a locally Nash stable $n$-jurisdiction structure if $g \in[\underline{g}(n), \bar{g}(n)]$ and $g \neq \frac{1}{2 n^{2}}$. Again, if $g=\frac{1}{2 n^{2}}$, the condition (24) in Lemma A.5 is violated, since the only case for $k>\frac{n}{2}$ is when $k=3$ for $n=4$, and $k=4,5$ when $n=6$. All cases 
can be easily examined, and a heterogeneous Nash locally stable $n$-jurisdiction structure fails to exist.

Proof of (iv). Let $n$ be either 5 or greater or equal to 7 . The analysis proceeds like in (iii) with the only difference that condition (24) is satisfied for $k=\frac{n}{2}+1$ or $k=\frac{n+1}{2}$ respectively, depending on the oddity of $n$, which yields the existence of a locally Nash stable heterogeneous $n$-jurisdiction structure for all $g \in[\underline{g}(n), \bar{g}(n)] . \square$

Proof of Proposition 4.5: (i) From the fact that $s \geq \hat{g}$ in any locally Nash stable heterogeneous structure we deduce that $\bar{s} \leq 3 \hat{g}$. Hence, in any locally Nash stable heterogeneous $n$ structure we must have $H(P) \leq 3$. To attain this value, take a $(n-1,1)$ structure for $g=\frac{3}{2(3 n-2)^{2}}$, where the size of the small jurisdictions is equal to $\hat{g}$. Hence, the size of the large one is equal to $3 \hat{g}$.

(ii) First we show that for every number of large coalitions $l=n-k$, there exists a value of $g$ such that a union of $k$ small and $n-k$ big jurisdictions is a locally Nash stable structure with small jurisdictions of the size $\hat{g}$. To find such a value of $g$, we solve the equation:

$$
k \hat{g}+3 l \hat{g}=1 \Leftrightarrow g=\frac{3}{2(n+2 l)^{2}} .
$$

We note that the maximal variance among the heterogeneous locally Nash stable $n$-structures is attained when the size of small jurisdictions is equal to $\hat{g}$. Indeed, consider an arbitrary locally Nash stable structure $P$, and let the size of small coalitions, $\underline{s} \neq \hat{g}$, or $H(P)<3$. This implies both $\underline{s}>\hat{g}$ and $\bar{s}<3 \hat{g}$. Thus, the variance is smaller than in the case where $\underline{s}=\hat{g}$ and $\bar{s}=3 \hat{g}$.

Thus, it suffice to examine the structures $P$ such that $H(P)=3$ for different $k, l$ such that $k s+3 l s=1$. From $s=\frac{1}{k+3 l}=\frac{1}{n+2 l}$ and $\bar{s}=\frac{3}{n+2 l}$, we are left with the following problem:

$$
\max _{l=1, \ldots, n-1}(n-l) \frac{1}{(n+2 l)^{2}}+l \frac{9}{(n+2 l)^{2}} \Leftrightarrow \max _{l=1, \ldots, n-1} \frac{n+8 l}{(n+2 l)^{2}} .
$$

Ignoring the integer constraint on $l$, this leads to $l=\frac{n}{4}$, and therefore $k=\frac{3 n}{4}$ small jurisdictions. This implies that the maximal variance is equal to $3 n /\left(n+\frac{n}{2}\right)^{2}-\frac{1}{n}=\frac{1}{3 n}$. If $n$ is not a multiple of 4 , the maximal variance is reached for one of the two integer values of $l$ close to $\frac{n}{4}$. 


\section{References}

Alesina, A., Baqir, R. and C. Hoxby (2004), Political jurisdictions in heterogeneous communities, Journal of Political Economy 112, 348-396.

Alesina, A. and E. Spolaore (1997), On the number and size of nations, Quarterly Journal of Economics 113, 1027-1056.

Aumann, R.J. (1959), Acceptable points in general cooperative $n$-person games, in Contributions to the Theory of Games, Vol IV, Tucker, A.W. and R.D. Luce, eds., Princeton University Press, Princeton.

Aumann, R.J. and J. Drèze (1974), Cooperative games with coalition structure, International Journal of Game Theory 3, 217-237.

Bogomolnaia, A. and M.O. Jackson (2002), The stability of hedonic coalition structures, Games and Economic Behavior 18, 201-230.

Bogomolnaia, A., Le Breton, M., Savvateev, A. and S. Weber (2005), Stable jurisdiction structures under equal share and median rules, CORE Discussion Paper \#2005/32.

Bogomolnaia, A., Le Breton, M., Savvateev, A. and S. Weber (2006), Stability under unanimous consent, free mobility and core, CORE Discussion Paper \#2006/7.

Demange, G. (1994), Intermediate preferences and stable coalition structures, Journal of Mathematical Economics 23, 45-58.

Eeckhout, J. (2004), Gibrat's Law for (All) Cities, American Economic Review 94, 1429-1451.

Ellison, G. and D. Fudenberg (2003), Knife-edge or plateau: when do market models tip?, Quarterly Journal of Economics 116, 1249-1278.

Gabaix, X. 1999, Zipf' law for cities: an explanation, Quarterly Journal of Economics 114, 739767.

Greenberg, J. (1994), Coalition structures, in Handbook of Game Theory with Applications, Vol. 2, Aumann, R. and S. Hart, eds., North Holland, Amsterdam.

Greenberg, J. and S. Weber (1986), Strong Tiebout equilibrium under restricted preferences 
domain, Journal of Economic Theory 38, 101-117.

Greenberg, J. and S. Weber (1994), Stable coalition structures in consecutive games, in Frontiers in Game Theory, Binmore, K., Kirman, A. and P. Tani, eds., MIT Press, Cambridge.

Guesnerie, R. and C. Oddou (1981), Second best taxation as a game, Journal of Economic Theory $25,67-91$

Guesnerie, R. and C. Oddou (1988), Increasing returns to size and their limits, Scandinavian Journal of Economics 90, 259-273.

Haimanko, O., Le Breton, M. and S. Weber (2005), Transfers in a polarized country: bridging the gap between efficiency and stability, Journal of Public Economics, forthcoming.

Haimanko, O., Le Breton, M. and S. Weber (2004), Voluntary formation of communities for the provision of public projects, Journal of Economic Theory 115, 1-34.

Jéhiel, P. and S. Scotchmer (1997), On the optimal number of jurisdictions, Annales d'Economie et de Statistique 45, 219-231.

Jéhiel, P. and S. Scotchmer (2001), Constitutional rules of exclusion in jurisdiction formation, Review of Economic Studies 68, 393-413.

Le Breton, M. and S. Weber (2003), The art of making everybody happy: how to prevent a secession, IMF Staff Papers 50, 403-435.

Mas-Colell, A. (1980), Efficiency and decentralization in the pure theory of public goods, Quarterly Journal of Economics 94, 625-641.

Rose, A. (2005), Cities and countries, CEPR Discussion Paper \# 5235.

Westhoff, F. (1977), Existence of equilibria in economies with a local public good, Journal of Economic Theory 17, 84-112.

Wooders, M.H. (1978), Equilibria, the core, and jurisdiction structures in economies with a local public good, Journal of Economic Theory 18, 328-348.

Wooders, M.H. (1980), The Tiebout hypothesis: near optimality in local public good economies, Econometrica 48, 1467-1486. 